\title{
When acoustic sequences are not perceptual sequences: The global perception of auditory patterns
}

\author{
RICHARD M. WARREN and JAMES A. BASHFORD, JR. \\ University of Wisconsin, Milwaukee, Wisconsin
}

\begin{abstract}
Warren, Bashford, and Gardner (1990) found that when sequences consisting of $1040-\mathrm{msec}$ steady-state vowels were presented in recycled format, minimal changes in order (interchanging the position of two adjacent phonemes) produced easily recognizable differences in verbal organization, even though the vowel durations were well below the threshold for identification of order. The present study was designed to determine if this ability to discriminate between different arrangements of components is limited to speech sounds subject to verbal organization, or if it reflects a more general auditory ability. In the first experiment, 1040 -msec sinusoidal tones were substituted for the vowels; it was found that the easy discrimination of minimal changes in order is not limited to speech sounds. A second experiment substituted 1040 -msec frozen noise segments for the vowels. The succession of noise segments formed a 400-msec frozen noise pattern that cannot be considered as a sequence of individual sounds, as can the succession of vowels or tones. Nevertheless, listeners again could discriminate between patterns differing only in the order of two adjacent 40 -msec segments. These results, together with other evidence, indicate that it is not necessary for acoustic sequences of brief items (such as phonemes and tones) to be processed as perceptual sequences (that is, as a succession of discrete identifiable sounds) for different arrangements to be discriminated. Instead, component acoustic elements form distinctive "temporal compounds," which permit listeners to distinguish between different arrangements of portions of an acoustic pattern without the need for segmentation into an ordered series of component items. Implications for models dealing with the recognition of speech and music are discussed.
\end{abstract}

A striking change in the mode of perception occurs when the duration of items in repeated sequences of steady-state vowels is below the 100 -msec threshold for identification of temporal order. From 30 to $100 \mathrm{msec} / \mathrm{item}$, the component vowels lose their identity, and the sequences are heard as illusory syllables and words consisting of consonants and vowels not present in the stimulus (Warren, Bashford, \& Gardner, 1990). Different arrangements of the vowels produce different verbal forms, allowing permuted orders of vowels to be recognized. The ability to distinguish between different arrangements is such that minimal changes in sequences consisting of $1040-\mathrm{msec}$ vowels can be detected: When the positions of two contiguous vowels were interchanged, listeners distinguished between the sequences on the basis of the different words heard (Warren et al., 1990).

This observation conflicts with models of speech recognition requiring both the identification and the ordering of individual speech sounds at an early stage in perceptual processing. For example, Jones (1978) stated,

This research as supported by Grant $88-0320$ from the Air Force Office of Scientific Research and Grant DC 00208 from the National Institute on Deafness and Other Communication Disorders. Correspondence should be addressed to R. M. Warren, Department of Psychology, University of Wisconsin, Milwaukee, WI 53201.
Music and speech are fast-paced auditory patterns that occur at rates above eight events per second, so it is clear that somehow people preserve order amongst successive sounds with durations in the neighborhood of $100 \mathrm{msec}$. But it is important to try to understand what occurs at these rates in order to understand how and when serial order of rapidfire sequences is preserved. (p.20)

Miller and Eimas (1982) stated clearly their conviction that phonetic segmentation plays a basic role in speech perception:

When a listener attends to speech, he or she perceives (although not always at a level of conscious awareness) a sequence of distinct and ordered units, the phonetic segments of language-roughly the consonants and vowels. A critical component of language comprehension, then, is the initial analysis of the waveform which yields a sequence of ordered phonetic segments. (p. 111) [italics added]

Were this so, and if speech perception were based upon an initial phonetic analysis of the waveform, then it is difficult to understand how sequences consisting of only brief steady-state vowels played loudly and clearly would be perceived as syllables that not only follow higher order phonotactic rules for phoneme clustering but also are restricted to syllables that actually occur in English (Chalikia \& Warren, 1991). 
Is the ability to distinguish minimal changes in order within complex sequences of brief sounds a unique linguistic skill limited to phonetic components, or is it a more general ability applicable to complex sequences of other sounds? In order to answer this question, in Experiment 1 of the present study, tones were substituted for the vowels employed by Warren et al. (1990). The listeners were presented with sequences of $1040-\mathrm{msec}$ sinusoidal tones, with each tone having a distinctive pitch when heard in isolation. The listeners attempted to distinguish between pairs of sequences differing only in the order of two contiguous items. As will be shown, they could distinguish between such pairs of 10-tone sequences, even though the 40 -msec duration of components was well below the threshold for direct identification of order in tonal sequences (Thomas \& Fitzgibbons, 1971) and well below the duration of notes required for the recognition of familiar melodies (Warren, Gardner, Brubaker, \& Bashford, 1991).

How can the different arrangements within phonetic sequences and tonal sequences be discriminated at item durations below the threshold for order identification? Warren (1982, pp. 129-138) reviewed evidence suggesting that permuted arrangements of sounds can be discriminated on the basis of global characteristics of the temporal pattern of neural stimulation without the need for resolution into component sounds. This concept considers that acoustic sequences of brief items need not function as perceptual sequences (i.e., they need not be processed as a succession of discrete sounds). If this theory concerning the basis for perception of speech and other acoustic sequences is correct, then a succession of individual sounds is not required for recognition of acoustic patterns, and interchanging sections of stochastic waveforms (frozen noises) should produce discriminable changes analogous to those resulting from interchanging orders of individual sounds within sequences. Experiment 2 tested this theory by substituting 10 frozen noise segments for the $1040-\mathrm{msec}$ tonal segments employed in Experiment 1. When these 40-msec noise fragments were concatenated and repeated, a 400-msec iterated noise pattern was created that lacked discrete components and hence could not be considered a sequence. Repeated frozen noise segments of this duration are heard as a repetitive "whooshing"' (Guttman \& Julesz, 1963). As will be shown, when such patterns were altered by interchanging positions of two contiguous $40-\mathrm{msec}$ fragments, listeners could discriminate between the two resulting patterns even though segmentation into an ordered sequence of discrete components was not possible.

\section{EXPERIMENT 1 \\ Discriminating Minimal Order Differences Within Ten-Item Sequences}

\section{Method}

Participants. Four listeners were employed. Listeners B.B. and J.B. had considerable experience as subjects in psychoacoustic ex- periments and had participated in preliminary observations with 10item sequences. Listeners J.R. and K.R. were untrained and had no prior experience with the stimuli employed in this study.

Stimuli and Apparatus. The 10-item sequences of sinusoidal tones used in Experiment 1 were synthesized using a Data Precision Co. Polynomial Waveform Synthesizer Model 2020-100 (40-kHz sampling frequency with 12-bit resolution). A pool of 48 randomly selected recycled tonal sequences (out of factorial 9 possible orderings) was constructed by sampling without replacement from a catalog of 10 sinusoidal frequencies ranging from 500 to $1500 \mathrm{~Hz}$ in equal logarithmic steps $(500,565,638,721,815,921,1040$, 1175,1328 , and $1500 \mathrm{~Hz}$ ). Linear ramps of $2.5 \mathrm{msec}(0 \mathrm{~dB}$ minimum) were imposed upon the onset and offset of each 40-msec tone burst for suppression of transients, and the amplitude envelopes of the bursts were scaled so that each would play back at the same level measured in dBA. For each of the original $48 \mathrm{~A}$ sequences, a single minimally contrasting $B$ sequence was created by reversing the order of two randomly selected contiguous tones.

Procedure. The listeners were tested individually in an audiometric room, with stimuli delivered at $70 \mathrm{dBA}$ through diotically wired TDH-49P headphones mounted in MX 41A/R cushions. Following pilot observations, an ABX procedure was selected, which facilitated accurate performance. A three-button panel was used by the listeners for switching between the contrasting $A$ and $B$ stimuli and a third, X, stimulus that was identical to the sequence presented in either the A or the B channel. They were permitted to switch at will between the three signals until they decided upon a match. The response of either " $A$ " or " $B$ " was recorded, along with the time required for the judgment. Trial-by-trial feedback concerning matching accuracy was provided.

The listeners participated in a total of 16 sessions, with each session lasting about 20 min and involving judgments of six contrasting sequences. Across the 16 sessions, the 48 contrasting pairs of sequences were presented twice to each listener for a total of 96 judgments. Each listener received a different random ordering of stimuli for his or her initial judgment for each of the contrasting pairs of sequences, and this order was repeated for that listener upon second presentation of the stimuli. Thus, the two judgments for each contrast were separated by judgments of the other 47 sequence pairs.

\section{Results}

The number of correct responses and median response times for judgments of the $\mathbf{4 8}$ pairs of tonal sequences are presented in Table 1. As can be seen, overall matching accuracy was well above chance for all listeners $(z \geq$ $7.76, p<.0001$ ), with the percentage of correct responses ranging from about $90 \%$ to $98 \%$. Judgments made by the experienced Listeners B.B. and J.B. were more accurate overall than were those of the untrained Listeners J.R. and K.R. $(z=3.23, p<.002)$.

Despite very high performance (i.e., ceiling effects) in Experiment 1, there was a reliable inverse correlation $[R=-.398, F(1,46)=8.64, p<.01]$ between the average proportion of correct discriminations and response time for the various tonal sequences when response times were averaged across listeners and log transformed. Log mean response times for the two trials presented for each sequence were also reliably correlated $[R=.66, F(1,46)=$ $36.32, p<.0001]$, indicating that there was substantial consistency in difficulty (about $44 \%$ of the variance in common) across first and second judgments of the specific tonal patterns. In order to determine the pattern attribute(s) responsible for this consistency in difficulty, an 
Table 1

Accuracy and Response Times for ABX Judgments

of Recycled 10-Tone Sequences ( $A$ and $B$ Sequences

Differed in the Order of a Single, Contiguous Pair of Tones)

\begin{tabular}{lccccc}
\hline & \multirow{2}{*}{$\begin{array}{c}\text { Number Correct } \\
\text { Listener }\end{array}$} & Percent & \multicolumn{3}{c}{ Response Time (seconds) } \\
\cline { 5 - 7 } B.B. & 94 & Correct & Median & Q1 & Q3 \\
J.B. & 94 & 97.9 & 25.0 & 15.5 & 37.5 \\
J.R. & 86 & 97.9 & 21.5 & 15.0 & 33.5 \\
K.R. & 87 & 89.6 & 89.5 & 45.0 & 161.5 \\
\hline N.R & 90.6 & 66.0 & 40.5 & 119.0 \\
\hline
\end{tabular}

Note-Accuracy scores for all listeners exceeded chance $(z \geq 7.76, p<.0001)$.

examination was made of the 48 sequences, involving all pairwise comparisons of the absolute frequency differences between the permuted tones and the immediately adjacent tones. Multiple regression analysis incorporating these frequency differences revealed that approximately $72 \%$ of the variance in $(\log )$ group mean response times across sequences could be accounted for by two factors [Multiple $R=0.85, F(2,45)=56.8, p<.0001$ ]: Times for judgments decreased with increasing frequency separation of the permuted tones $\left(t_{\beta 1}=7.73, p<.0001\right)$, and also decreased with increasing frequency separation between the tones immediately preceding and following the permuted pair $\left(t_{\beta 2}=6.08, p<.0001\right)$.

\section{Discussion}

It has been recognized for some time that some sequences with permuted arrangements of items can be discriminated as global entities having emergent Gestalt characteristics that differ for different arrangements. Ladefoged and Broadbent (1959) and Efron (1963) described this holistic pattern recognition for a sequence of two sounds presented as AB or BA. Garner and his associates (Garner \& Gottwald, 1967, 1968; Royer \& Garner, 1970; Royer \& Robin, 1986) described the organizational rules governing global pattern recognition for complex patterns consisting of two brief easily discriminated sounds (such as Tone A and Tone B), which were used to generate repeated patterns (for example, the recycled sequence $A B A A B B A B A A B B A B A . .$. , in which the repeating unit is nine items long). Watson and his colleagues have reported a number of experiments examining the ability of listeners to discriminate changes within "word-length" sequences consisting of 10 sinusoidal tones presented as unrecycled single statements (see Watson, 1987, for review). They were interested primarily in the effects of changes in a single tone (such as its intensity, frequency, and duration) rather than differences in the temporal order of unchanged components, as in the present study. However, Watson and Kelly (1981) concluded that when listeners were familiar with the tonal pattern and the position of the individual item subject to possible change, judgments appeared to involve "wholepattern features," which then provided access to stored details concerning the target sound. In the studies by Watson's group, the ability to detect changes in the one-shot (nonrecycled) sequences required weeks and sometimes months of training. The techniques of recycling complex sequences employed by Garner's group and in the present study greatly facilitated performance, and, as shown in Table 1, accurate discrimination took place within a few minutes or less.

Much of the interest in perception of tonal and other nonverbal sequences results from its assumed relevance to mechanisms employed for the perception of speech. This is reflected not only by Watson's choice of the term word length for his tonal sequences, but by attempts by a number of investigators to relate receptive aphasia and dysphasia to high thresholds for the identification of order of tones, hisses, and buzzes (Bond, 1976; Brookshire, 1972; Carmon \& Nachshon, 1971; Tallal \& Piercy, 1973, 1974). However, there is a problem in relating thresholds for the identification of order to disorders in speech perception: thresholds are too high even for normal listeners. The average duration of phonemes in normal speech can be as low as $70 \mathrm{msec}$, and some intelligibility is still possible with artificially "compressed" speech at $30 \mathrm{msec} / \mathrm{phoneme}$ (see Warren, 1982, pp. 119-120). When the special ease of identifying the initial and terminal sounds of sequences is minimized or eliminated by appropriate experimental procedures, then the threshold for order identification is roughly $150 \mathrm{msec}$ for sequences of heterogeneous sounds, such as hisses and tones (Warren \& Ackroff, 1976), about $125 \mathrm{msec}$ for sequences consisting solely of tones (Thomas \& Fitzgibbons, 1971), and about $100 \mathrm{msec}$ for speech sounds (Dorman, Cutting, \& Raphael, 1975; Thomas, Cetti, \& Chase, 1971).

When listeners are not required to identify the order of tones, but only to recognize and discriminate between different arrangements, the task can be accomplished at much shorter item durations (Nickerson \& Freeman, 1974; Warren \& Byrnes, 1975; Warren et al., 1991). The ability to distinguish between different arrangements of brief nonverbal sounds is not restricted to tonal sequences. When recycled sequences consisted of different arbitrarily selected items (e.g., a sinusoidal tone, a square wave, and a noise), it was possible for listeners to distinguish between the different arrangements of items down to $5 \mathrm{msec} /$ item (Warren, 1974). This study also provided further evidence for an important caveat first suggested by Ladefoged and Broadbent (1959) and by Efron (1963) on the basis of their experiments with two-item sequences: Once listeners are familiar with contrasting orders and 
are provided information, either directly or indirectly, concerning the nature and arrangement of constituent elements, then it is easy for them to provide an accurate analytic description by rote. Thus, under these conditions, order identification does not precede, but follows the prior recognition of the overall pattern.

It appears that there are two fundamentally different ways of discriminating between permuted arrangements of the same sounds, and much confusion in the literature has resulted from a failure to differentiate between the ability to identify components and their orders and the ability to recognize patterns holistically. Melodic recognition is an especially interesting case. The duration between the successive notes of melodies ranges from about $150 \mathrm{msec}$ to $1 \mathrm{sec}$ (Fraisse, 1957/1963), and it has been found that the recognition of repeated familiar melodies is limited to roughly the same range-when notes were played at rates either below or above these limits, recognition was not possible (Warren et al., 1991). At rates slower than about $1.3 \mathrm{sec} /$ note, listeners could not identify the melodies unless they were familiar with musical notation; at rates faster than about $160 \mathrm{msec} /$ note, literacy in musical notation made no difference, and all listeners were unable to identify the tunes. However, at these fast rates, it was observed that although the melodies could not be identified, the notes seemed to form distinctive nonmelodic patterns. This observation was verified in Experiment 1 of the present study, in which the 40 -msec duration of the individual tones was well below the threshold for order identification. The listeners could detect minor changes in arrangement, so that when the order of two contiguous items was reversed, they could distinguish between the two orders. This conflicts with the claim of Winckel (1967) that when tones are below the durational threshold for order identification, a perceptual "metathesis" occurs and different orders become indistinguishable. It appears instead that rapid sequences of tones form "temporal compounds," with different arrangements forming discriminable compounds, as suggested by Warren (1974).

Is it possible that what appears to be a globally organized temporal compound is actually based upon an initial analysis into an ordered series of tones at a level below "conscious awareness," as suggested by Miller and Eimas (1982) for speech? While it is difficult to refute the hypothesis that the order of individual tones is resolved and utilized at an inaccessible level, it is possible to determine whether or not an analysis into an ordered series of discrete sounds is required for the recognition of, and discrimination between, different arrangements of brief components. In order to make this determination, patterns were constructed that could not be considered as a succession of discrete sounds and, hence, were not sequences.

In Experiment 2, 10 40-msec frozen noise segments were substituted for the $1040-\mathrm{msec}$ tones in Experiment 1 . Again, contrasting pairs were created by interchanging the positions of two adjacent 40 -msec sections.
However, when the 10 noise segments were joined end to end, they formed a single 400 -msec frozen noise lacking an internal structure of discrete components. If different arrangements of contiguous portions of the frozen noise pattern could be discriminated, listeners could not be basing their responses upon an initial segmentation into an ordered series of identifiable elements.

\section{EXPERIMENT 2 \\ Discriminating Minimal Order Differences Within Ten-Item Patterns Consisting of Frozen Noise Segments}

\section{Method}

Participants. The four listeners employed in Experiment 1 were also employed in Experiment 2.

Stimuli and Apparatus. For synthesis of the 10 -item sequences of reproducible frozen noises used in Experiment 2, a Data Precision Model 6100 Universal Waveform Analyzer, operating at a sampling rate of $40 \mathrm{kHz}$ with 14-bit resolution, was used to excise 10 40-msec segments of on-line Gaussian noise produced by a Brüel and Kjaer Type 1405 generator. These 10 segments were sampled randomly without replacement and concatenated in digital form to create 48 10-item sequences, and contrasting $A$ and $B$ versions of each were produced by permuting the order of a single pair of contiguous $40-\mathrm{msec}$ items. As was done with the tone bursts employed in Experiment 1, linear onset and offset ramps of $2.5 \mathrm{msec}$ were used for suppression of transients.

Digital-to-analog conversion and playback of the tone and noise sequences in recycling form were accomplished using the Data Precision waveform synthesizer, operating at a sampling frequency of $40 \mathrm{kHz}$ with 12-bit resolution. The analog playback of the recycling sequences was recorded on an Otari Model MTR 90-II 16track recorder, with the sequences $A, B$, and $X$ (which could be $A$ or $B$ ) recorded in parallel on three separate tracks. During the experiments, the output of the recorder was amplified by a Neotek Series I audio mixer, and then bandpass filtered from $50 \mathrm{~Hz}$ to $8000 \mathrm{~Hz}$ with slopes of $115 \mathrm{~dB} /$ octave (Wavetek/Rockland Model 751A Brickwall filter).

Procedure. The procedure was identical to that employed in Experiment 1 .

\section{Results and Discussion}

The listeners readily detected recycling of the 400 -msec frozen noise patterns. They reported hearing the repetitive whooshing that was described by Guttman and Julesz (1963) for iterated frozen noise segments having periods from $250 \mathrm{msec}$ to $1 \mathrm{sec}$. In addition, a complex assortment of repetitive transient sounds, such as "bumps," "beeps," and "clanks," emerged after the initial undifferentiated whooshing was heard. Within a few minutes, the listeners could usually find patterns that differed for contrasting pairs of sequences.

The number of correct responses and the median response time for judgments of the $\mathbf{4 8}$ pairs of frozen noise sequences are given in Table 2 . The table shows that overall matching accuracy for sequences of frozen noise segments was well above chance for all listeners $(z \geq 4.79$, $p<.0001$ ), with the percentage of correct responses ranging from about $80 \%$ to $98 \%$ correct. As was found with tonal sequences in Experiment 1, judgments made 
Table 2

Accuracy and Response Times for ABX Judgments of Recycled 10-Item Sequences of Frozen Noise Segments ( $A$ and $B$ Sequences Differed in the Order of a Single, Contiguous Pair of Noise Segments

\begin{tabular}{lccccc}
\hline & \multirow{2}{*}{$\begin{array}{c}\text { Number Correct } \\
\text { (Out of 96) }\end{array}$} & Percent & \multicolumn{2}{c}{ Response Time (seconds) } \\
\cline { 5 - 6 } Listener & Correct & Median & Q1 & Q3 \\
\hline B.B. & 94 & 97.9 & 71.5 & 41.5 & 123.0 \\
J.B. & 94 & 97.9 & 55.5 & 37.0 & 93.5 \\
J.R. & 77 & 80.2 & 193.0 & 89.0 & 340.5 \\
K.R. & 84 & 87.5 & 111.0 & 58.0 & 252.0 \\
\hline Note-Accuracy scores for all listeners exceeded chance $(z \geq 5.92, p<.0001)$
\end{tabular}

Note-Accuracy scores for all listeners exceeded chance $(z \geq 5.92, p<.0001)$.

by the experienced Listeners B.B. and J.B. were significantly more accurate $(z>4.79, p<.0001)$ than those of the inexperienced Listeners J.R. and K.R.

Discriminating minimal order contrasts within the novel complex patterns formed by concatenated segments of frozen noise required more time than did the analogous task involving tonal sequences (group median time per trial $89 \mathrm{sec}$ vs. $39 \mathrm{sec}$, respectively). However, the accuracy of discrimination for the two types of patterns was numerically identical for the 2 experienced listeners (98\%); the largest difference, obtained for Listener J.R. (89.6\% correct for tones vs. $80.2 \%$ correct for noise), was not statistically significant $(z=1.81, p>.05)$.

Regression analysis of $\log$ response times across first and second judgments of the noise sequences provided evidence of intrasubject consistency in pattern difficulty for 3 of the 4 listeners $[R=0.34$ to $0.47, F(1,46)>$ $5.91, p<.02$ or better]. However, response times were not correlated across listeners, suggesting that perceptual organization of iterated frozen noise may be quite idiosyncratic, in keeping with both the variety and lability of perceptual organizations reported for individual frozen noise patterns.

Warren and Bashford (1981) described informal observations indicating that when three 50 -msec frozen noise segments designated as $\mathrm{A}, \mathrm{B}$, and $\mathrm{C}$ were concatenated and recycled, listeners could readily discriminate between the 150 -msec recycled noises consisting of $(\mathrm{ABC})_{n}$ and $(\mathrm{ACB})_{n}$. This task was much easier than the task in the present Experiment 2: Listeners in the earlier study immediately perceived a clear difference in the two 150 -msec recycled noises (the 150-msec repetition period of the frozen noise was in the "motorboating" range of 50$250 \mathrm{msec}$ described by Guttman \& Julesz, 1963). The motorboating patterns sounded homogenous and differed from each other in timbre. This contrasted with the whooshing patterns in Experiment 2, which were characterized by labile component features changing in richness and variety over time. Also, only three frozen noise segments or "items" were joined to form the patterns used in the earlier study. Thus, when the order was altered, the relative position of each section shifted relative to each of the other two $(A \rightarrow B \rightarrow C \rightarrow A$... vs. $A \rightarrow C \rightarrow B \rightarrow A .$.$) . In Experiment 2, the order of only$ two contiguous $40-\mathrm{msec}$ sections of the $400-\mathrm{msec}$ stochas- tic pattern were interchanged, so that the relative positions of most of the sections remained fixed $(A \rightarrow B \rightarrow C \rightarrow D \rightarrow$ $\mathrm{E} \rightarrow \mathrm{F} \rightarrow \mathrm{G} \rightarrow \mathrm{H} \rightarrow \mathrm{I} \rightarrow \mathrm{J} \rightarrow \mathrm{A} \ldots$ vs. $\mathrm{A} \rightarrow \mathrm{B} \rightarrow \mathrm{C} \rightarrow \mathrm{D} \rightarrow \mathrm{F} \rightarrow \mathrm{E} \rightarrow$ $\mathrm{G} \rightarrow \mathrm{H} \rightarrow \mathrm{I} \rightarrow \mathrm{J} \rightarrow \mathrm{A} \ldots$..).

\section{GENERAL DISCUSSION}

Earlier studies have shown that when positional cues involving the initial and terminal items in sequences are eliminated or minimized by recycling, the threshold for identifying the order of components is quite high$100 \mathrm{msec}$ or more depending upon the types of sounds and the response procedures. However, it also has been reported that a different kind of discrimination can permit recognition of different arrangements of components as global patterns called temporal compounds when items are too brief to permit identification of order. Warren et al. (1990) found that when sequences of steady-state vowels having durations between 30 and $100 \mathrm{msec}$ were recycled, the component vowels lost their identity and formed temporal compounds consisting of syllables and words. These verbal forms could be used to discriminate between phonetic sequences having minimal differences in temporal order, so that when two contiguous $40-\mathrm{msec}$ vowels in 10-item sequences had their positions interchanged, then the sequences could be discriminated on the basis of different verbal organizations.

In Experiment 1 of the present study, sinusoidal tones were substituted for the vowels used in the previous investigation, and it was found that minimal differences in the temporal order of components (interchanging the positions of two contiguous 40 -msec tones in a 10 -item recycled sequence) produced discriminable changes. It is suggested that sequences of brief items form globally organized patterns that permit discrimination of permuted arrangements. This ability is not restricted to humans. There have been reports that different temporal arrangements of brief sounds can be discriminated by monkeys, dolphins, and cats (for a review and discussion of this literature, see Warren, 1988). Furthermore, in the case of cats, there is direct evidence that the response of auditory neurons to a specific tone changes with the tone's position within a sequence, so that discriminating different arrangements would not necessarily require segmentation into an ordered sequence of components. Weinberger and 
McKenna (1988) and McKenna, Weinberger, and Diamond (1989) reported that when complex sequences of tones were presented to "awake" cats, recordings from the primary (AI) and secondary (AII) cortex showed that the response to individual tones depended upon the presence and position of other tones. In both of these papers, it was suggested that these findings were related to Warren's concept of "wholistic" pattern recognition of sequences by humans.

In Experiment 2, segments of frozen noise were substituted for the 1040 -msec tones. When the noise segments were concatenated, they formed a single $400-\mathrm{msec}$ pattern. The patterns formed by linking random waveforms were not sequences-they did not consist of a succession of discrete acoustic elements, as did the tones in Experiment 1 . Nevertheless, the listeners were able to discriminate between patterns produced by interchanging the position of two adjacent $40-\mathrm{msec}$ segments with the same accuracy found for tonal sequences.

These observations indicate that sequences of brief items, whether speech sounds, tones, or stochastic waveforms derived from noise, can be processed globally as complex patterns or temporal compounds, and that resolution into discrete components is not required for discriminating between different arrangements of the acoustic components. We suggest that it is misleading to consider acoustic sequences of brief items (such as the phonemes in speech) as perceptual sequences, and that the models of speech perception involving analyses into component phonetic segments may be inappropriate.

\section{REFERENCES}

Bond, Z. S. (1976). On the specification of input units in speech perception. Brain \& Language, 3, 72-87.

BROOKSHIRE, R.H. (1972). Visual and auditory sequencing by aphasic subjects. Journal of Communication Disorders, 5, 259-269.

CARMON, A., \& NaCHSHON, I. (1971). Effect of unilateral brain damage on perception of temporal order. Cortex, 7, 410-418.

Chalikia, M. H., \& Warren, R. M. (1991). Phonemic transformations: Mapping the illusory organization of steady-state vowel sequences. Language \& Speech, 34, 109-143.

Dorman, M. F., Cutting, J. E., \& Raphael, L. J. (1975). Perception of temporal order in vowel sequences with and without formant transitions. Joumal of Experimental Psychology: Human Perception \& Performance, 104, 121-129.

EFroN, R. (1963). Temporal perception, aphasia, and déjà vu. Brain, 86, 403-424.

Fraisse, P. (1963). The psychology of time (J. Leith, Trans.). New York: Harper \& Row. (Original work published 1957)

GaRner, W. R., \& GotTWaLD, R. L. (1967). Some perceptual factors in the learning of sequential patterns of binary events. Journal of Verbal Learning \& Verbal Behavior, 6, 582-589.

Garner, W. R., GotTwald, R. L. (1968). The perception and learning of temporal pattems. Quarterly Journal of Experimental Psychology, 20, 97-109.

GuTTMAN, N., \& Julesz, B. (1963). Lower limit of auditory periodicity analysis. Joumal of the Acoustical Society of America, 35, 610.

JONES, M. R. (1978). Auditory patterns: Studies in the perception of structure. In E. C. Carterette \& M. P. Friedman (Eds.), Handbook of perception: Vol. 8, Perceptual coding (pp. 255-288). New York: Academic Press.
Ladefoged, P., \& Broadbent, D. E. (1959). Perception of sequence in auditory events. Quarterly Journal of Experimental Psychology, $12,162-170$.

McKenna, T. M., Weinberger, N. M., \& Diamond, D. M. (1989). Responses of single auditory cortical neurons to tone sequences. Brain Research, 481, 142-153.

Miller, J. L., \& Eimas, P. D. (1982). Feature detectors and speech perception: A critical evaluation. In D. G. Albrecht (Ed.), Recognition of pattern and form (pp. 111-145). New York: Springer-Verlag.

Nickerson, R. S., \& FreEMAN, B. (1974). Discrimination of the order of the components of repeating tone sequences: Effects of frequency separation and extensive practice. Perception \& Psychophysics, 16, 471-477.

Royer, F. L., \& Garner, W. R. (1970). Perceptual organization of nine-element auditory temporal patterns. Perception \& Psychophysics, 7, 115-120.

RoYer, F. L., \& RobIN, D. A. (1986). On the perceived unitization of repetitive auditory patterns. Perception \& Psychophysics, 39, 9-18.

Tallal, P., \& Piercy, M. (1973). Defects of nonverbal auditory perception in children with developmental aphasia. Nature, 241, 468-469.

Tallal, P., \& PIERCy, M. (1974). Developmental aphasia: Rate of auditory processing and selective impairment of consonant perception. Neuropsychologia, 12, 83-93.

Thomas, I. B., Cetti, R. P., \& Chase, P. W. (1971). Effect of silent intervals on the perception of temporal order for vowels. Journal of the Acoustical Society of America, 49, 84. (Abstract)

Thomas, I. B., \& Fitzgibbons, P. J. (1971). Temporal order and perceptual classes. Journal of the Acoustical Society of America, 50, 8687. (Abstract)

WARREN, R. M. (1974). Auditory temporal discrimination by trained listeners. Cognitive Psychology, 6, 237-256.

WARREN, R. M. (1982). Auditory perception: A new synthesis. Elmsford, NY: Pergamon.

WARREN, R. M. (1988). Perceptual bases for the evolution of speech. In M. E. Landsberg (Ed.), The genesis of language: A different judgment of evidence (pp. 101-110). Berlin: Mouton de Gruyter.

WARREN, R. M., \& ACKROFF, J. M. (1976). Two types of auditory sequence perception. Perception \& Psychophysics, 20, 387-394.

WARren, R. M., \& BASh Ford, J. A., JR. (1981). Perception of acoustic iterance: Pitch and infrapitch. Perception \& Psychophysics, 29, 395-402.

Warren, R. M., Bashford, J. A., JR., \& Gardner, D. A. (1990). Tweaking the lexicon: Organization of vowel sequences into words Perception \& Psychophysics, 47, 423-432.

WARREN, R. M., \& ByrNES, D. L. (1975). Temporal discrimination of recycled tonal sequences: Pattern matching and naming of order by untrained listeners. Perception \& Psychophysics, 18, 273-280.

Warren, R. M., Gardner, D. A., Brubaker, B. S., \& Bashford, J. A., JR. (1991). Melodic and nonmelodic sequences of tones: Effects of duration on perception. Music Perception, 8, 277-290.

WATSON, C. S. (1987). Uncertainty, informational masking, and the capacity of immediate memory. In W. A. Yost \& C. S. Watson (Eds.), Auditory processing of complex sounds (pp. 267-277). Hillsdale, NJ: Erlbaum.

WATSON, C. S., \& KELLY, W. J. (1981). The role of stimulus uncertainty in the discrimination of auditory patterns. In D. J. Getty \& J. H. Howard (Eds.), Auditory and visual pattern recognition (pp. 37-59). Hillsdale, NJ: Erlbaum Press.

Weinberger, N. M., \& MCKenNa, T. M. (1988). Sensitivity of single neurons in auditory cortex to contour: Toward a theory of neurophysiology of music perception. Music Perception, 5, 355-390.

WINCKEL, F. (1967). Music, sound and sensation: A modern exposition. New York: Dover.

(Manuscript received October 22, 1991; revision accepted for publication December 31, 1992.) 